\title{
Modelling free surface flows with smoothed particle hydrodynamics
}

\author{
L.Di G.Sigalotti, J.Daza, A.Donoso \\ Centro de Física, Instituto Venezolano de Investigaciones Científicas, IVIC, \\ Apartado Postal 21827, Caracas 1020A, Venezuela
}

Received March 9, 2006, in final form April 17, 2006

In this paper the method of Smoothed Particle Hydrodynamics (SPH) is extended to include an adaptive density kernel estimation (ADKE) procedure. It is shown that for a van der Waals (vdW) fluid, this method can be used to deal with free-surface phenomena without difficulties. In particular, arbitrary moving boundaries can be easily handled because surface tension is effectively simulated by the cohesive pressure forces. Moreover, the ADKE method is seen to increase both the accuracy and stability of SPH since it allows the width of the kernel interpolant to vary locally in a way that only the minimum necessary smoothing is applied at and near free surfaces and sharp fluid-fluid interfaces. The method is robust and easy to implement. Examples of its resolving power are given for both the formation of a circular liquid drop under surface tension and the nonlinear oscillation of excited drops.

Key words: gas-liquid and vacuum-liquid interfaces, surface tension and related phenomena, heat conduction, computational methods in fluid dynamics

PACS: $68.03 .-g, 68.03 . C d, 44.10 .+i, 47.11 .+j$

\section{Introduction}

Gas-liquid flows are examples of typical two-phase fluids encountered in many systems within both natural and artificial environments, where the liquid is separated from the gas by a free surface whose shape is determined by the flow field. In particular, liquid surfaces are known to be in a state of tension because fluid molecules at or near the surface experience uneven forces of attraction [1]. Hence surface tension is the result of a microscopic, localized force that exerts itself on the fluid elements at the surface in both the normal and tangential directions. Examples of free-surface motion induced by tension forces can be found in a variety of flows which are either natural or artificially constructed. For instance, well-known problems involving liquid-gas and liquid-vacuum interfaces result from experiments and studies of droplet dynamics, which may include the formation and evaporation of equilibrium liquid drops $[2,3]$, the nonlinear oscillations of excited drops [4-7], the breakup of liquid filaments [8,9] and the coalescence of colliding drops [10-15].

In general, the detailed analysis of free-surface flows involves the use of numerical methods coupled to appropriate boundary conditions to simulate an arbitrarily moving surface. However, most of the available methods suffer from difficulties in modelling topologically complex interfaces having surface tension. Among the earlier numerical schemes, the MAC method [16] was known to be the most flexible and robust. It relies on finite differences to solve the hydrodynamic equations and on particles to define the surface. The method was further extended to deal with moving boundaries [17] and applied to a wide variety of problems, including waterfalls, breaking dams and two-fluid instabilities [18]. In spite of attempts to simplify it, it remains complicated to program. Later on, Brackbill et al. [19] developed the continuum-surface-force (CSF) method, which treats surface tension as a continuous, three-dimensional effect across the interface rather than as a pure boundary condition. In this model, the interfaces between fluids of different properties, or colours,

*E-mail: lsigalot@cassini.ivic.ve 
are represented as transition layers of finite thickness, across which the colour variable varies continuously. Although this method has been successfully applied to simulate fluid phenomena involving incompressible fluid flow in low-gravity environments, capillarity and droplet dynamics, it still requires the use of complicated surface-tracking techniques, like the MAC method, to accurately model the volume force along the surface curvature. Alternative surface-tracking methods have been proposed that rely on the spine-flux technique [20]. However, a serious drawback of these latter methods is that they are limited to surface deformations that do not result in double-definition of the surface along a spine. On the other hand, an application of the CSF model to calculate interfacial curvatures with Smoothed Particle Hydrodynamics (SPH) was reported by Morris [21]. Moreover, Monaghan [22] showed that SPH can be easily extended to handle free-surface phenomena if the continuity equation, rather than the usual summation interpolant, is used in calculating the density and if the boundary particles are allowed to move with a corrected velocity.

Here we present an alternative approach to model free-surface flows under tension forces with standard SPH. The method is modified to account for an adaptive density kernel estimation (ADKE) procedure similar to that described by Silverman [23]. In this procedure the basic kernel and nearest neighbour SPH approaches are combined in a way that the amount of applied smoothing is minimized in regions where the density is lower. As a result, near a free surface, where there is a crippling deficiency of particles, the ADKE method uses much smaller kernels than in the bulk of the fluid without compromising the SPH stability, leading to a finer resolution of the free surface compared to other SPH schemes. Coupled to the fully Lagrangian nature of SPH, the ADKE method maintains very well sharp fluid-fluid interfaces and free surfaces with no need of employing explicit surface reconstruction. The method was also found to give stable and accurate results for supersonic compressible flows with strong shocks [24]. In this paper, we focus on two-dimensional test calculations of the formation of circular drops under surface tension and its nonlinear oscillations starting from elongated shapes. The paper is organized as follows. In section 2 we write down the SPH hydrodynamic equations that are solved and provide a brief description of the method. Then in section 3 we describe the results of a few model calculations and in section 4 we give the conclusions.

\section{SPH equations and numerical method}

In contrast to Monaghan's [22] approach, the density $\rho_{a}$ of the particles is computed using the summation interpolant

$$
\rho_{a}=\sum_{b=1}^{N} m_{b} W_{a b},
$$

where $m_{b}$ is the mass of particle $b, W_{a b}=W\left(\left|\mathbf{x}_{a}-\mathbf{x}_{b}\right|, h\right)$ is a spherically symmetric kernel function and $h$ is the so-called smoothing length or width of the kernel. In this work, we adopt the quartic spline kernel of Lucy [25] so that only nearest particles within a circle of radius $h$ contribute to the SPH summation in equation (1). The same kernel was used by Nugent and Posch [2] in SPH simulations of drop condensation. Other smoothing kernels, such as the cubic $B$-spline [26] or the quintic spline [21], have been used by other authors. Test simulations of drop condensation with the quartic and quintic spline kernels have shown that the results are almost independent of the form of the kernel. For the SPH equations of motion and thermal energy we use a symmetrized representation that contains the full Newtonian viscous stress tensor $S^{i j}$ and the Fourier's heat flux vector $q^{i}$, namely

$$
\begin{aligned}
\frac{\mathrm{d} x_{a}^{i}}{\mathrm{~d} t} & =v_{a}^{i}, \\
\frac{\mathrm{d} v_{a}^{i}}{\mathrm{~d} t} & =\sum_{b=1}^{N} m_{b}\left(\frac{S_{a}^{i j}}{\rho_{a}^{2}}+\frac{S_{b}^{i j}}{\rho_{b}^{2}}\right) \frac{\partial W_{a b}}{\partial x_{a}^{j}}, \\
\frac{\mathrm{d} U_{a}}{\mathrm{~d} t} & =\frac{1}{2} \sum_{b=1}^{N} m_{b}\left(\frac{S_{a}^{i j}}{\rho_{a}^{2}}+\frac{S_{b}^{i j}}{\rho_{b}^{2}}\right)\left(v_{b}^{i}-v_{a}^{i}\right) \frac{\partial W_{a b}}{\partial x_{a}^{j}}-\sum_{b=1}^{N} m_{b}\left(\frac{q_{a}^{j}}{\rho_{a}^{2}}+\frac{q_{b}^{j}}{\rho_{b}^{2}}\right) \frac{\partial W_{a b}}{\partial x_{a}^{j}},
\end{aligned}
$$


where $x_{a}^{i}$ denotes the position of the particles, $v_{a}^{i}$ is their velocity and $U_{a}$ is their thermal energy per unit mass. The viscous stress tensor is given by

$$
S^{i j}=-p \delta^{i j}+\eta\left(\frac{\partial v^{i}}{\partial x^{j}}+\frac{\partial v^{j}}{\partial x^{i}}\right)+\left(\zeta-\frac{2}{d} \eta\right) \frac{\partial v^{k}}{\partial x^{k}} \delta^{i j},
$$

and the heat flux vector is defined according to

$$
q^{i}=-\kappa \frac{\partial T}{\partial x^{i}} .
$$

In these relations, $p$ is the internal (isotropic) pressure, $T$ is the fluid temperature, $\delta^{i j}$ is the unit tensor, $\eta$ and $\zeta$ are the coefficients of shear and bulk viscosity, $\kappa$ is the coefficient of heat conduction and $d$ is a number equal to 2 or 3 depending on whether the motion is confined to twoor three-dimensions, respectively.

For the drop model of this paper, we assume a van der Waals (vdW) fluid, where the pressure and thermal energy obey the constitutive relations

$$
\begin{aligned}
p & =\frac{\rho \bar{k}_{\mathrm{B}} T}{1-\rho \bar{b}}-\bar{a} \rho^{2}, \\
U & =\frac{\xi}{2} \bar{k}_{\mathrm{B}} T-\bar{a} \rho .
\end{aligned}
$$

In these equations, $\xi$ is the number of degrees of freedom of the molecules and $\bar{k}_{\mathrm{B}}=k_{\mathrm{B}} / m$, where $k_{\mathrm{B}}$ is the Boltzmann's constant and $m$ is the particle mass. Furthermore, $\bar{a}=a / m^{2}$ and $\bar{b}=b / m$, where $a$ controls the strength of the attractive forces between the molecules and $b$ is a constant due to their finite size.

As was first noted by Nugent and Posch [2], surface tension effects can be simulated with the aid of equation (7) by separating the cohesive term, $-\bar{a} \rho^{2}$, from the remainder forces. The same applies to the energy term, $-\bar{a} \rho$, in equation (8). The former term contributes with an attractive central force between the SPH particles, which produces a net acceleration given by

$$
\frac{\mathrm{d} v_{a}^{i}}{\mathrm{~d} t}=2 \bar{a} \sum_{b=1}^{N} m_{b} \frac{\partial W_{a b}^{H}}{\partial x_{a}^{i}},
$$

while the latter term contributes with an effective heating due to the work done by the cohesive pressure forces on the liquid within the free surface and takes the SPH form

$$
\frac{\mathrm{d} U_{a}}{\mathrm{~d} t}=2 \bar{a} \sum_{b=1}^{N} m_{b}\left(v_{b}^{i}-v_{a}^{i}\right) \frac{\partial W_{a b}^{H}}{\partial x_{a}^{i}} .
$$

In these equations $W_{a b}^{H}=W\left(\left|\mathbf{x}_{a}-\mathbf{x}_{b}\right|, H\right)$ has the same functional form of the interpolating kernel employed for all other terms, except that it now depends on the smoothing range $H$ instead of $h$. In the CSF model, surface tension is translated into a force per unit volume, $\mathbf{F}_{s}=\mathbf{f}_{s} \delta_{s}$, where $\delta_{s}$ is a normalized function, which peaks at the interface, and $\mathbf{f}_{s}$ is a force per unit area defined as

$$
\mathbf{f}_{s}=\sigma \tau \mathbf{n}+\nabla_{s} \sigma,
$$

where $\sigma$ is the surface tension coefficient, $\mathbf{n}$ is the unit normal to the interface, $\tau$ is the curvature of the interface and $\nabla_{s}$ is the surface gradient. Note that the first term in equation (11) is a force acting normal to the interface and corresponds to the net surface tension force due to the local curvature. This force acts to smooth regions of high curvature, in an attempt to reduce the total surface area and hence the surface energy. In contrast, the second term in equation (11) acts tangentially to the interface, forcing fluid elements on the surface to move from regions of low surface tension to regions of higher surface tension. The surface normal in equation (11) is evaluated 
according to $\mathbf{n}=\nabla c /[c]$, where $c$ is the colour function identifying each fluid in the problem and $[c]$ is the jump in $c$ across the interface. Moreover, the curvature is calculated using the relation $\tau=-\nabla \cdot \mathbf{n}$, whereas $\delta_{s}=|\mathbf{n}|$. In SPH form, Morris [21] suggested the following representations

$$
\mathbf{n}_{a}=\sum_{b=1}^{N} \frac{m_{b}}{\rho_{b}} c_{b} \nabla_{a} W_{a b},
$$

and

$$
(\nabla \cdot \mathbf{n})_{a}=\sum_{b=\mathbf{1}}^{N} \frac{m_{b}}{\rho_{b}} \mathbf{n}_{b} \cdot \nabla_{a} W_{a b}
$$

for the normal and the surface curvature, respectively. In particular, the divergence of the surface normal is proportional to the surface tension force. We note the intrinsic similarity between equations (9) and (13), implying that the former acts effectively as a true surface tension force due to the local curvature. It then follows that the cohesive term in equation (7) is all we need to correctly model surface tension in a vdW fluid. Furthermore, the choice of $H$ in equations (9) and (10) is determined by the same considerations that led to a substantial improvement of the interface stability properties with the CSF method employed by Morris [21]. In practice, stability is maintained when $H \geqslant 2 h$. In a fluid bounded by a free surface, the forces represented by equation (9) largely cancel within the bulk of the fluid, except for a small strip $H$ around the surface where particles are accelerated in the direction of the inward surface normal. In actual numerical simulations, the value of $H$ determines the thickness of the interface.

Apart from showing how the vdW cohesive pressure can be used with SPH to model free-surface phenomena we show why the ADKE procedure is an essential ingredient to maintain both adequate stability and fine resolution of the interface. The essence of the ADKE method is to construct a collection of local kernels at the position of the particles in order to allow the smoothing length to vary from point to point. In order to do so, an initial, or pilot, density estimate, $\hat{\rho}_{a}$, is calculated using the summation interpolant in equation (1) with $W_{a b}=W\left(\left|\mathbf{x}_{a}-\mathbf{x}_{b}\right|, h_{0}\right)$, where $h_{0}$ is defined as some dilation factor of the initial interparticle separation, i.e., $h_{0}=D \Delta s$. Local bandwidth factors, $\lambda_{a}$, are next calculated according to

$$
\lambda_{a}=k\left(\frac{\hat{\rho}_{a}}{\bar{g}}\right)^{-\varepsilon},
$$

where $\bar{g}$ is the geometric mean of the pilot estimates given by

$$
\log \bar{g}=\frac{1}{N} \sum_{b=1}^{N} \log \hat{\rho}_{b},
$$

$k$ is a scaling factor of the order of unity and $\varepsilon$ is the sensitivity parameter defined in the interval $0 \leqslant$ $\varepsilon \leqslant 1$. The adaptive estimator is finally constructed by recalculating the density using equation (1) with $h_{0}$ replaced by $h_{a}=\lambda_{a} h_{0}$. As long as $\varepsilon \rightarrow 1$, the $h_{a}$ 's become more sensitive to the density distribution, implying a greater difference between the smoothing length in different parts of the fluid domain. This means that near a sharp interface, where there is a crippling deficiency of particles, the size of the kernels will be much smaller than in the bulk of the fluid, leading to a finer resolution of the interface in spite of the fact that $H \geqslant 2 h$ in order to ensure numerical stability. Moreover, setting $\varepsilon=0, \lambda_{a} \rightarrow k$ and the ADKE method reduces to the usual fixed width kernel approach. Also, note that with the $\lambda_{a}$ 's calculated as in equation (14), the $h_{a}$ 's effectively control the scale of smoothing applied to the data, thus improving the accuracy and stability near the interface.

\section{Numerical tests}

In this section, we consider a series of two-dimensional simulations for the specific case of a forming vdW liquid drop under surface-tension forces, in which the effects of viscosity and heat 
conduction are taken into account. This model represents a sensitive test calculation to quantify the resolving power of the ADKE method on free-surface flows. Its ability to handle arbitrarily moving surfaces is also tested against the nonlinear oscillations of an initially deformed drop.

\subsection{Formation of equilibrium vdW drops}

We first consider the formation of a stable, circular drop using the same vdW parameters employed by Nugent and Posch [2] and Meleán et al. [3], namely $m=1, \bar{a}=2, \bar{b}=0.5$ and $\bar{k}_{\mathrm{B}}=1$. In these reduced units, the critical point of the vdW fluid occurs for $\rho_{c r}=2 / 3, p_{c r}=8 / 27$ and $T_{c r}=32 / 27$ [27]. For simplicity, all variables are normalized with respect to their critical values. The coefficients of thermal conductivity, shear and bulk viscosity in reduced units are chosen to be $\kappa=5, \eta=1$ and $\zeta=0.1$, respectively. In addition, the outer medium is a vacuum so that there is no heat conduction between the drop and its surroundings. All calculations start with $900 \mathrm{SPH}$ particles of equal mass $\left(m_{a}=m=1\right)$ distributed at the vertices of a square cell array. Initially the particles are at rest and separated from one another by a distance $\Delta s=0.75$ along the $x$ - and $y$-axes. The choice of these parameters is such that the initial density, as calculated using equation (1), satisfies the constraint $\rho_{0}<1 / \bar{b}$. Each particle is given an initial temperature $T_{0}=0.2$ so that the inequality $\bar{k}_{\mathrm{B}} T_{0}>2 \bar{a} \rho_{0}\left(1-\rho_{0} \bar{b}\right)^{2}$ is also satisfied for thermodynamic stability. At this subcritical temperature, an equilibrium, circular drop with no external atmosphere is expected to form [2]. Therefore, no periodic boundary conditions are required far away from the initial square array of particles. All runs were made by setting $H=2 h$ in equations (9) and (10).

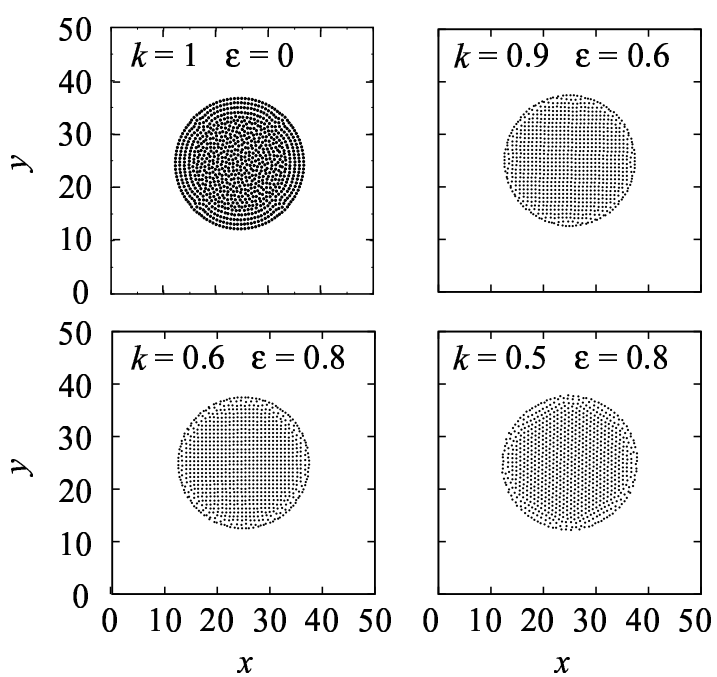

Figure 1. Particle positions in the $(x, y)$-plane showing the structure of a circular liquid drop, with $D=3, k=1$ and $\varepsilon=0$ (top left panel); $D=3$, $k=0.9$ and $\varepsilon=0.6$ (top right panel); $D=4$, $k=0.6$ and $\varepsilon=0.8$ (bottom left panel) and $D=5$, $k=0.5$ and $\varepsilon=0.8$ (bottom right panel).

We consider three distinct sequences of computations, all starting with identical initial conditions. Each sequence is defined by fixing the value of $D$ and varying the ADKE parameters $k$ and $\varepsilon$. In particular, we choose $D=3,4$ and 5 so that initially $h_{0}=D \Delta s$, and values of $k$ and $\varepsilon$ in the intervals $[0.5,1.0]$ and $[0.5,0.9]$, respectively. A typical evolution proceeds as follows. At the beginning, the internal pressure is not enough to balance the surface tension forces, causing an inward motion of the particles at and near the corners of the square boundary. As a result, part of the surface tension energy is converted into internal liquid movement with a consequent sharp increase of the kinetic energy. Circularization of the confined liquid is approximately completed when about 95 percent of the kinetic energy is dissipated by viscous forces. During this stage, the thermal energy also increases due to the heating produced by internal friction near the drop surface. The increase of thermal energy soon slows down due to heat conduction. The further evolution consists of a very slow decrease of the kinetic energy accompanied by an equally slow increase of the thermal energy until the drop reaches thermo-mechanical equilibrium with a circular shape, as shown in figure 1 . With a constant timestep $\Delta t=0.005$, a typical evolution is completed after about 130,000 computational cycles.

The four panels of figure 1 show the final equilibrium drop as obtained for different choices of the ADKE parameters. The top left panel corresponds to the case when the ADKE procedure is dropped (i.e., $k=1, \varepsilon=0$ ). Since for this model the dilation factor is $D=3$, the calculation was made using a fixed kernel approach of width $h=3 \Delta s$, resulting in $H=6 \Delta s=4.5$. We may see 

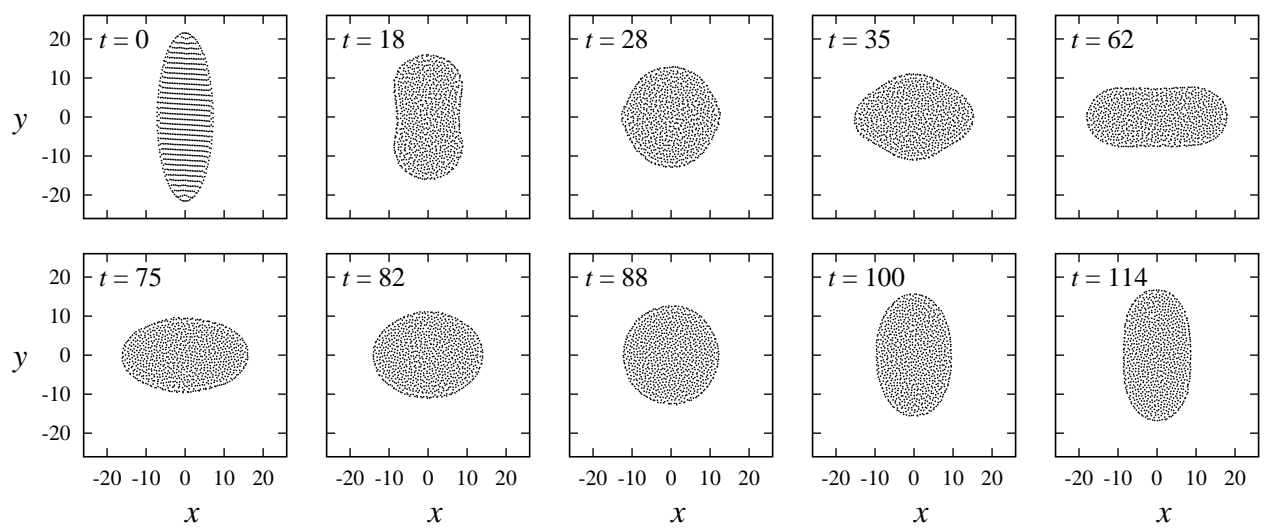

Figure 2. Sequence of times showing the first period of oscillation of a liquid drop when it is released from an elliptic shape with aspect ratio $a / b=3(t=0)$ at $\operatorname{Re} \approx 522$. In each panel the time is shown in reduced units.

that in this case the transition layer separating the drop from the outer vacuum is considerably thicker compared to the other three models where the ADKE procedure was activated. In fact, the drop surface is numerically resolved by the outermost four concentric circles of particles of thickness $\approx 4.5$. In all other cases where the ADKE was used the outer interface is clearly represented by only one outer circle of particles. The much better resolution afforded in these latter cases is a consequence of both the adaptive nature of the method and the fact that a minimum smoothing is effectively applied to the data at the drop surface.

\subsection{Oscillating vdW drops}

Next, we test the response of the method on large-amplitude oscillations of vdW drops that are released from a static elliptic shape. A sequence of equilibrium circular drops was first constructed with the same parameters as before, except that the shear viscosity was varied in the range $0.012 \leqslant$ $\eta \leqslant 12$, corresponding to Reynolds number in the interval $0.5<\operatorname{Re}<522$. In all cases, the initial smoothing length was chosen to be $h_{0}=3 \Delta s$, while the ADKE parameters were fixed to $k=0.9$ and $\varepsilon=0.6$. The reference circular drops were deformed into an elliptic shape by homogeneous flattening under pure shear strain [28], which is achieved with an area-preserving and, hence, density-conserving transformation of the particle coordinates

$$
x^{\prime}=\frac{1}{(1+\epsilon)} x, \quad y^{\prime}=(1+\epsilon) y,
$$

where $\epsilon$ is the elongation. The above transformation converts the circle into an ellipse of semi-major axis $a$ aligned with the $y$-axis, as shown in the first panel of figure 2. Here we shall consider only drop deformations with an initial aspect ratio $a / b=3$, where $b$ denotes the semi-minor axis of the ellipses.

The nonlinear oscillations of a liquid drop represent a more stringent test because they involve a moving free surface. The time resolved evolution for the case of a drop with $\operatorname{Re} \approx 522(\eta=0.012)$ is displayed in figure 2 during its first period of oscillation. The elongated drop first contracts along its major axis as most of its surface energy is being transformed into internal liquid movement. It then passes through a transient circular shape $(t=28)$ before reaching a stage of maximum elongation along the $x$-axis $(t=62)$. At this point, most of the undamped internal kinetic energy is in the form of surface energy. The rim pressure soon exceeds the stagnation pressure inside the drop, causing it to contract back (along the $x$-axis) under surface tension. The drop evolves again to an approximate circular shape before achieving a prolate shape towards the completion of the first oscillation period at $t=114$. At this time, the aspect ratio is $\approx 1.94$ and decays further to $\approx 1.35$ by the end of the second period $(t=221)$. The oscillatory behaviour will continue in 
time with progressively lower amplitudes until eventually the drop loses all of its internal kinetic energy by viscous dissipation. The evolution was followed for about 20 periods by the time the drop has returned to its equilibrium circular shape. The damping of the oscillations is mostly due to viscous dissipation and, to some extent, to the finite heat conductivity $(\kappa=5)$. A value of $\kappa$ this large serves to reduce temperature gradients and density fluctuations within the drop. However, the intrinsic viscosity which is inherent to particle systems due to the smoothing may also contribute to dissipation of the drop oscillations even when $\eta=0$. A test simulation with $\eta=0$ and $\kappa=0$ shows that by switching off the ADKE procedure, the rate of dissipation due to the inherent numerical viscosity is much slower than that due to either finite physical viscosity or heat conductivity. For comparison, the ADKE method (with $k=0.9$ and $\varepsilon=0.6$ ) resulted in even slower dissipation rates, mainly because much less smoothing was actually needed at and near the drop surface. Evidently, these results show that the method is able to handle moving surfaces without difficulties. Another important feature is that during surface motion the boundary particles tended to move from regions of low curvature to regions of higher curvature, responding to the action of the tangential component of the surface tension force.

\section{Conclusions}

We have performed test calculations which show that standard SPH can be used to accurately simulate free-surface flows without difficulty for a van der Waals (vdW) fluid, provided that the kernel function is modified to include an adaptive density kernel estimation (ADKE) procedure, like the one proposed by Silverman [23]. When dealing with a vdW-type equation of state, the SPH representation of the cohesive pressure gradients strongly resembles that employed to calculate interfacial curvatures with SPH using the continuum-surface-force model [21], implying that surface tension is correctly simulated by the cohesive part of the vdW pressure. The ADKE procedure increases the resolving power of SPH and provides a satisfactory representation of free boundaries. In particular, the ADKE method constructs local bandwidth factors, or kernel estimates, evaluated at the position of particles such that the width of the interpolating kernel is allowed to vary from point to point. This results in much smaller sizes of the kernel close to sharp boundaries or in regions where the density is lower, implying that the amount of smoothing required in those regions is effectively minimized. This is the key point which allows for a much better description of free surfaces and sharp fluid-fluid interfaces than the fixed width kernel approach does. Here the method has been successfully tested for a forming two-dimensional, circular drop under the effects of surface tension and for the two-dimensional oscillations of an initially elongated drop about a circular shape. The method is simple to implement and does not require special modifications when applied to fully three-dimensional problems.

\section{Acknowledgements}

This work is financially supported by the Instituto Venezolano de Investigaciones Científicas (IVIC). One of us (L. Di G. S.) acknowledges the Fondo Nacional de Ciencia, Tecnología e Innovación (Fonacit) of Venezuela for partial support.

\section{References}

1. Nelleon M. Mechanics and Properties of Matter. Heineman, London, 1952.

2. Nugent S., Posch H.A., Phys. Rev. E, 2000, 62(4), 4968.

3. Meleán Y., Sigalotti L.Di G., Hasmy A., Comput. Phys. Commun., 2004, 157, 191.

4. Basaran O.A., J. Fluid Mech., 1992, 194, 169.

5. Becker E., Hiller W.J., Kowalewski J., Fluid Mech., 1994, 258, 191.

6. Apfel R.E. et al., Phys. Rev. Lett., 1997, 78(10), 1912.

7. Mashayek F., Ashgriz N., Phys. Fluids, 1998, 10(5), 1071.

8. Schulkes R.M.S.M., J. Fluid Mech., 1996, 309, 277.

9. Notz P.K., Basaran O.A., J. Fluid Mech., 2004, 512, 223. 
10. Jiang Y.J., Umemura A., Law C.K., J. Fluid Mech., 1992, 234, 171.

11. Qian J., Law C.K., J. Fluid Mech., 1997, 331, 59.

12. Menchaca-Rocha A., Huidobro F., Martinez-Davalos A., Michaelian K., Perez A., Rodriguez V., Cârjan N., J. Fluid Mech., 1997, 346, 291.

13. Willis K.D., Orme M.E., Exp. Fluids, 2000, 29, 347.

14. Mashayek F., Ashgriz N., Minkowycz W.J., Shotorban B., Int. J. Heat Mass Transfer, 2003, 46, 77.

15. Meleán Y., Sigalotti L.Di G., Int. J. Heat Mass Transfer, 2005, 48, 4041.

16. Harlow F., Welch J.E., Phys. Fluids, 1965, 9, 842.

17. Viecelli J., J. Comput. Phys., 1971, 8, 119.

18. Harlow F., Comput. Phys. Commun., 1988, 48, 1.

19. Brackbill J.U., Kothe D.B., Zemach C., J. Comput. Phys., 1992, 100, 335.

20. Mashayek F., Ashgriz N., J. Comput. Phys., 1995, 122, 367.

21. Morris J.P., Int. J. Numer. Meth. Fluids, 2000, 33, 333.

22. Monaghan J.J., J. Comput. Phys., 1994, 110, 399.

23. Silverman B.W. Density Estimation for Statistics and Data Analysis. Chapman \& Hall, London, 1996.

24. Sigalotti L.Di G., López H., Donoso A., Sira E., Klapp J., J. Comput. Phys., 2006, 212, 124.

25. Lucy L.B., Astron J., 1977, 80, 1013.

26. Monaghan J.J., Lattanzio J.C., Astron. Astrophys., 1985, 149, 135.

27. Landau L.D., Lifshitz E.M. Statistical Physics. Addison-Wesley, Massachusetts, 1969.

28. Twiss R.J., Moores E.M. Structural Geology. Freeman, New York, 1992.

\title{
Моделювання вільних поверхневих потоків із згладженою частинковою гідродинамікою
}

\author{
Л.Ді Г.Сігалотті, Х.Даса, А.Доносо \\ Центр фізики, Венесуельський інститут наукових досліджень, \\ пошт. скринька 21827, Каракас 1020-А, Венесуела
}

Отримано 9 березень 2006 р., в остаточному вигляді - 17 квітня 2006 р.

\begin{abstract}
Метод згладженої частинкової електродинаміки розширено для врахування процедури оцінки адаптивного ядра густини. Показано, що для ван-дер-Ваальсової рідини цей метод може без труднощів застосовуватися при описі явищ поблизу вільної поверхні. Зокрема, легко врахувати довільно рухомі поверхні, оскільки поверхневий натяг ефективно симулюється силами когезивного тиску. Більше того, точність і стійкість цього методу вища, оскільки він дозволяє локально змінювати ширину інтерполянти ядра так, що застосовується мінімальне необхідне згладжування поблизу вільних поверхонь і поверхонь розділу флюїд-Флюїд. Метод є стійким і легким у застосуванні. Приводяться приклади його використання для опису формування краплин рідини під впливом поверхневого натягу та нелінійного коливання збуджених крапель.
\end{abstract}

Ключові слова: поверхні розділу газ-рідина і вакуум-рідина, поверхневий натяг і пов 'язані явища, теплопров ідність, обчислювальні методи в динаміці флюїдів

PACS: 68.03.-g, 68.03.Cd, 44.10. $+i, 47.11 .+j$ 